\title{
Ultrasound improves the outcomes of cardiopulmonary resuscitation in rats by stimulating the cholinergic anti-inflammatory pathway
}

\author{
YUHAN ZHANG ${ }^{1,2^{*}}$, YUE SONG ${ }^{3,4^{*}}$, TINGTING SHU ${ }^{1,5}$, LICAI LIANG ${ }^{1}$, \\ WEIJING SHAO ${ }^{1}$, LANG GUO $^{1}$ and PENG SUN ${ }^{1}$ \\ ${ }^{1}$ Department of Emergency Medicine, Union Hospital, Tongji Medical College, \\ Huazhong University of Science and Technology, Wuhan, Hubei 430022; ${ }^{2}$ Department of Intensive Care Unit, \\ Xiangyang Central Hospital, Affiliated Hospital of Hubei University of Arts and Science, Xiangyang, \\ Hubei 441021; ${ }^{3}$ Department of Ultrasound and ${ }^{4}$ Hubei Province Key Laboratory of Molecular Imaging, \\ Union Hospital, Tongji Medical College, Huazhong University of Science and Technology, Wuhan, Hubei 430022; \\ ${ }^{5}$ Department of Intensive Care Unit, Wuhan Hospital of Traditional Chinese Medicine, Wuhan, Hubei 430010, P.R. China
}

Received January 30, 2019; Accepted July 2, 2019

DOI: $10.3892 / \mathrm{mmr} .2019 .10527$

\begin{abstract}
The present study investigated the effects of the ultrasound (US), a noninvasive technique, on ischemia-reperfusion injury (IRI) following cardiopulmonary resuscitation (CPR). The animals used in the present study were randomized into five groups ( $n=8$ per group) as follows: i) The CPR group, where the rats underwent $6 \mathrm{~min}$ of untreated ventricular fibrillation (VF) followed by CPR and defibrillation; ii) the US group, in which the treatment was identical to the CPR group with the exception that rats were exposed to US treatment $24 \mathrm{~h}$ prior to CPR; iii) the MLA group, in which the treatment was identical to the US group with the exception that the $\alpha 7$ nicotinic acetylcholine receptor $(\alpha 7 \mathrm{nAChR})$ antagonist MLA (4 mg/kg) was administered 30 min prior to US and VF respectively; iv) the GTS group, in which the treatment was identical to the CPR group with the exception that the $\alpha 7 \mathrm{nAChR}$ agonist GTS-21 (4 mg/kg) was injected $30 \mathrm{~min}$ prior to $\mathrm{VF}$; and v) the SHAM group, in which the rats were exposed to surgical preparation without CPR and US application. At 1 day prior to CPR, the US treatment was administered to the left kidney by US pulses (contrast general mode with $9 \mathrm{MHz}$ ) with a bursting mechanical index of 0.72 for $2 \mathrm{~min}$. Following treatment of the left kidney,
\end{abstract}

Correspondence to: Professor Peng Sun, Department of Emergency Medicine, Union Hospital, Tongji Medical College, Huazhong University of Science and Technology, 1277 Jiefang Avenue, Wuhan, Hubei 430022, P.R. China

E-mail: sp761397@sina.com

*Contributed equally

Key words: cardiac arrest, ultrasound, $\alpha$ nicotinic acetylcholine receptor, cholinergic anti-inflammatory pathway, neurological function the right kidney was exposed to identical US treatment for an additional $2 \mathrm{~min}$. The results demonstrated that US preconditioning decreased the number of defibrillations required and shortened the duration of CPR. US also suppressed tumor necrosis factor- $\alpha$ and interleukin- 6 levels following resuscitation $(\mathrm{P}<0.05)$, and a significantly longer overall survival time was observed in the US-treated animals $(\mathrm{P}<0.01)$. In addition, US attenuated neuronal injury and promoted the expression of $\alpha 7 \mathrm{nAChR}$ in hippocampal neurons $(\mathrm{P}<0.05)$. However, the protective effects of US were abolished by MLA and imitated by GTS-21. The results of the present study demonstrated that prior exposure to US may improve animal outcomes following $\mathrm{CPR}$, and the protective effects of US may be dependent on the cholinergic anti-inflammatory pathway (CAP) via $\alpha 7 n$ AChR.

\section{Introduction}

Patients who are admitted to the intensive care unit (ICU) following successful resuscitation have a poor prognosis and a high risk of postresuscitation disease, a condition that includes multiple life threatening disorders, including neurological failure. The mechanisms underlying postresuscitation disease have been suggested to involve whole-body ischemia and reperfusion syndrome, which triggers a systemic inflammatory response and is characterized by high levels of circulating cytokines and inflammatory mediators, similar to patients with sepsis (1). Therefore, strategies to limit the high levels of circulating cytokines and thereby improving patient outcomes following resuscitation have become a focus of an increasing number of studies.

Efferent signals transmitted by the vagus nerve via the neurotransmitter acetylcholine may attenuate local and systemic inflammation, and this regulatory activity has been termed 'the cholinergic anti-inflammatory pathway' (CAP) (2). Vagus nerve stimulation (VNS) may exert a protective effect against acute cerebral, cardiac and kidney ischemia reperfusion injury (IRI) by suppressing inflammation and 
apoptosis via the alpha 7 nicotinic acetylcholine receptor ( $\alpha 7 \mathrm{nAChR})$ (3-5). It has recently been demonstrated that VNS may improve survival in rat models of cardiopulmonary resuscitation (CPR) (6), indicating that VNS may be an alternative therapeutic strategy for CPR with a neural interface approach.

Despite preclinical evidence demonstrating the benefits of VNS for IRI, this therapy has not yet been implemented in a clinical setting. A major reason is the lack of an established technique that makes VNS easier to administer and less invasive in a clinical setting. In addition, numerous cholinergic pharmacologic agonists, including nicotine and acetylcholine exhibit a narrow therapeutic index, which limits their clinical use (7). A study by Gigliotti et al (8) described a non-pharmacological, noninvasive, ultrasound-based method to prevent renal IRI in mice. The results of this study demonstrated that a blockade or genetic deficiency of $\alpha 7 \mathrm{nAChR}$ abrogated the protective effect of ultrasound (US), suggesting the involvement of the CAP (8). This study highlighted the possibility of a novel approach to prevent IRI following CPR. Therefore, the aim of the present study was to determine whether US, as a clinical applicable noninvasive approach, would improve the outcomes following resuscitation in a CPR rat model. It was hypothesized that US would exert a protective effect against IRI induced by CPR predominantly through the activation of CAP via $\alpha 7 n A C h R$.

\section{Materials and methods}

Animal preparation. Male Sprague-Dawley rats (aged 13-16 weeks and weighing 350-400 g) were purchased from Experimental Animal Research Center of Hubei Province [Laboratory Animal Use Certificate NO. SCXK (E) 2015-0018] and were housed in a temperature-controlled room with a $12 \mathrm{~h}$ light/dark cycle. All animals had ad libitum access to standard food and water. All protocols in the animal model were conducted in strict accordance with the guidelines of the Ministry of Science and Technology of the People's Republic of China for Animal Care and Use, which conformed to the Guide for the Care and Use of Laboratory Animals published by the United States of America National Institutes of Health (9). The study was approved by the Medical Ethics Committee of Huazhong University of Science and Technology (approval no. 2017-S2191).

Experimental protocols. To determine the effect of US on survival following CPR, the rats were randomly assigned to one of five groups (n=8/group) as follows: i) The CPR group, where the rats underwent 6 min of untreated VF followed by CPR and defibrillation; ii) the US group, in which the treatment was identical to the CPR group, with the exception that rats were exposed to US $24 \mathrm{~h}$ prior to CPR; iii) the MLA group, in which the treatment was identical to the US group, with the exception that the $\alpha 7 \mathrm{nAChR}$ antagonist MLA ( $4 \mathrm{mg} / \mathrm{kg}$ ) was administered at $30 \mathrm{~min}$ prior to US and VF respectively; iv) the GTS group, in which the treatment was identical to the CPR group, with the exception that the $\alpha 7 \mathrm{nAChR}$ agonist GTS-21 (4 mg/kg) was injected at $30 \mathrm{~min}$ prior to VF; and v) the SHAM group, in which the rats were exposed to surgical preparation without CPR and US application. All animals were observed closely for $72 \mathrm{~h}$, following which they were euthanized with an intraperitoneal injection of sodium pentobarbital $(150 \mathrm{mg} / \mathrm{kg})$. At necropsy, the organs were inspected for gross abnormalities, including evidence of traumatic injuries resulting from cannulation, airway management or precordial chest compression.

US treatment protocol. A previous study demonstrated that US may activate CAP by stimulating the kidney (8). Therefore, the present study elected to perform the same US protocol as was performed in the study of Gigliotti et al (8). For US exposure, the rats were anesthetized with an intraperitoneal injection of pentobarbital sodium $(45 \mathrm{mg} / \mathrm{kg}) 1$ day prior to establishing the CA/CPR model. The rats were subsequently placed on the operating table in a supine position with their abdominal and back fur shaved. Pre-warmed ultrasound gel was placed on their depilated skin for US treatment. A clinical Philips IU Elite Ultrasound machine coupled with a L9-3 probe was used for US treatment. Firstly, the left kidney was localized using conventional B-mode image. Then, pulsed high power US was delivered to the left kidney (contrast general mode) under burst mode at a mechanical index of 0.72 . The US with a pulse length of $1 \mathrm{sec}$ was applied every $6 \mathrm{sec}$ for a total of $2 \mathrm{~min}$. The right kidney was subsequently exposed to US using the same procedure. Following US treatment, the animals were permitted to recover from the anesthetic in a temperature-controlled incubator.

Cardiac arrest (CA) model protocol. Rats were fasted overnight; however, they were permitted ad libitum access to water during the preparation period. The rats were anesthetized with an intraperitoneal injection of pentobarbital sodium $(45 \mathrm{mg} / \mathrm{kg})$. In order to maintain the depth of anesthesia, additional doses $(10 \mathrm{mg} / \mathrm{kg})$ were administered approximately once per hour. Following trachea intubation, the rats were mechanically ventilated with a tidal volume of $0.65 \mathrm{ml} / 100 \mathrm{~g}$, a respiratory rate of $70 / \mathrm{min}$, and a fraction of inspired oxygen $\left(\mathrm{FiO}_{2}\right)$ of 0.21 using the ALC-V8S ventilator (Shanghai Alcott Biotechnology Co., Ltd.). A conventional lead II ECG was continuously recorded by subcutaneous needle electrodes. PE50 catheters were inserted into the left femoral artery and vein in order to measure arterial pressure and to establish an intravenous infusion passage. Arterial blood pressure and ECG were recorded using the BL-420F data acquisition and analysis system (Chengdu Taimeng Software Co., Ltd.). The core temperature was monitored by a rectal temperature probe and maintained at $36.5 \pm 0.5^{\circ} \mathrm{C}$ during the experiment by a heating lamp and heating pad. The basal parameters of the rats were recorded $15 \mathrm{~min}$ prior to the induction of ventricular fibrillation (VF). VF was induced by a modified method based on transcutaneous electrical epicardium stimulation, as described previously (10). Electrical stimulation was performed using an electrical stimulator from the BL-420F Biofunctional Experimental System with current mode, continuous single stimulation pattern, a delay of $100 \mathrm{msec}$, a width of $1 \mathrm{msec}$, a frequency of $50 \mathrm{~Hz}$, an initial intensity of $1 \mathrm{~mA}$ and a stimulation time of $3 \mathrm{~min}$. A stimulation time of $3 \mathrm{~min}$ was selected in order to prevent spontaneous defibrillation. The non-intervention time was $3 \mathrm{~min}$. Therefore, the total cardiac arrest time was $6 \mathrm{~min}$. At $6 \mathrm{~min}$ following the initiation of VF, CPR was initiated. The mechanical ventilation was changed 


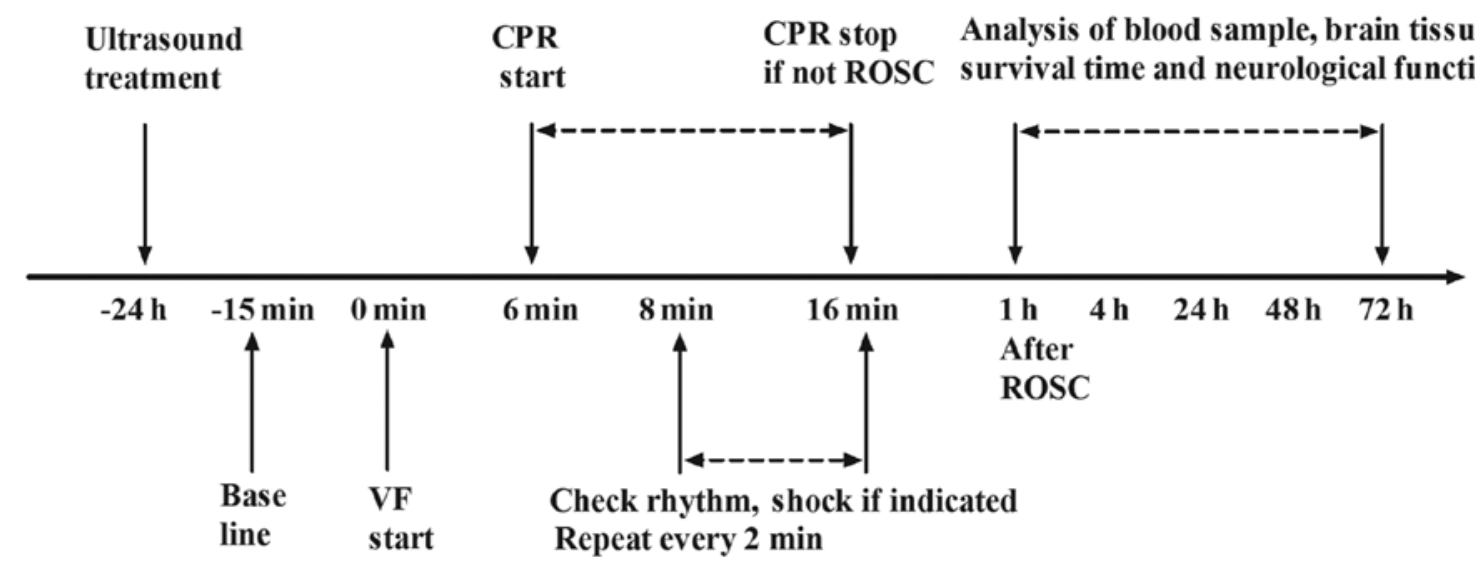

Figure 1. Experimental protocol. Ultrasound was applied to the rats 1 day prior to induction of VF. VF, ventricular fibrillation; ROSC, return of spontaneous circulation; CPR, cardiopulmonary resuscitation.

to a tidal volume of $0.65 \mathrm{ml} / 100 \mathrm{~g}$, a respiratory rate $(\mathrm{RR})$ of 100 times/min, and a $\mathrm{FiO} 2$ of 1.0. Chest compression was applied at a frequency of 200 times/min (with the assistant of a metronome). The depths of compressions were initially adjusted to maintain an aortic diastolic pressure between 26 and $28 \mathrm{mmHg}(11)$. Adrenaline $\{20 \mu \mathrm{g} / \mathrm{kg}$, [Grand Pharma (China) Co., Ltd.]\} was intravenously administered immediately following the initiation of CPR and was repeated once every $3 \mathrm{~min}$ if necessary. At $2 \mathrm{~min}$ after CPR, defibrillation was performed with $4 \mathrm{~J}$ if the ECG indicated VF. If the rats achieved restoration of spontaneous circulation (ROSC) with the return of supraventricular rhythm or a systolic pressure above $60 \mathrm{mmHg}$ for $10 \mathrm{~min}$ after defibrillation, the rescue measurements were stopped and investigators monitored the ECG and hemodynamic parameters. If not, CPR was repeated and defibrillation was performed again 2 min following CPR. If animals did not achieved ROSC after 10 min with the above treatment, CPR was terminated and this process was considered a failure. Following resuscitation the hemodynamic parameters were continuously monitored and recorded for $4 \mathrm{~h}$. All catheters, including the endotracheal tube, were removed at $4 \mathrm{~h}$ post-ROSC. Then animals were subsequently returned to their cages for close observation (Fig. 1).

Evaluation of neurological defect score (NDS). Neurological function was evaluated according to the method of NDS at $24 \mathrm{~h}$ intervals for a total of $72 \mathrm{~h}$. The neurological deficits were scored from 0 (no observed neurologic deficit) to 500 (mortality or brain death) (12). NDS was examined and confirmed by two independent investigators who were blinded to the study.

Measurement of serum tumor necrosis factor- $\alpha(T N F-\alpha)$ and interleukin-6 (IL-6) levels. Blood samples were obtained from the rats at 1,4 and $72 \mathrm{~h}$ post ROSC through the femoral vein. The samples were centrifuged at $2,963 \mathrm{x} \mathrm{g}$ at $4^{\circ} \mathrm{C}$ for $15 \mathrm{~min}$ and the supernatant was collected and then stored at $-80^{\circ} \mathrm{C}$. ELISAs were performed according to the manufacturers' protocol with rat-TNF- $\alpha$ Immunoassay (cat. no. RTA00; R\&D Systems Europe, Ltd.) and rat-IL-6 ELISA kit (cat. no. CSB-E04640r; Cusabio Technology LLC). Briefly, polystyrene 96-well microtitel immunoplates were coated with the affinity-purified polyclonal rat anti-TNF- $\alpha$ antibody. Then the samples and the TNF- $\alpha$ standard solutions were distributed in each plate, and the plates were washed and incubated with anti-TNF- $\alpha$ galactosidase for $2 \mathrm{~h}$ at room temperature. The plates were subsequently washed and incubated with substrate solution for $30 \mathrm{~min}$ at room temperature. Following this incubation, the optical density was measured using an ELISA reader. This method was also employed to examine the levels of IL-6 according to the aforementioned protocol and with relevant modifications.

Histopathological analysis. After 3 days, the rats were sacrificed and the hippocampus was rapidly removed from the cerebrum. The left half of the hippocampus was stored at $-80^{\circ} \mathrm{C}$ and used later for western blot analysis. The right half of the hippocampus was fixed in $4 \%$ paraformaldehyde overnight at $4^{\circ} \mathrm{C}$ and embedded in paraffin for immunohistochemical analysis. The present study focused on the hippocampal CA1 region, which is considered to be the most vulnerable region of the brain to ischemia and hypoxia. The slides were stained with hematoxylin and eosin: The sections were placed in $0.5 \%$ hematoxylin staining solution for $10 \mathrm{~min}$ at room temperature; then immersed in $0.5 \%$ eosin aqueous solution for $5 \mathrm{~min}$ at room temperature. Sections were rinsed with running water for 20 min after each step. Dead neurons were determined by the presence of hypereosinophilic cytoplasm and pyknotic nuclei. Nonviable neurons were counted in 3 random microscopic fields per section, and the percentage of nonviable neurons in 3 sections per animal was averaged (high-power fields of view, x200 magnification) using a light microscope (Olympus BX51; Olympus Corporation).

Immunofluorescence staining. The hippocampal slides were deparaffinized, rehydrated in a descending ethanol series $(100$, 95,85 and $75 \%$, and $\mathrm{ddH}_{2} \mathrm{O}$ ) and immersed in $3 \% \mathrm{H}_{2} \mathrm{O}_{2}$ /methanol for $10 \mathrm{~min}$ at room temperature to inactivate endogenous peroxidase activity. Antigens were heat-retrieved in sodium citrate buffer (10 mM sodium citrate; $0.05 \%$ Tween-20; pH 6.0) at $100^{\circ} \mathrm{C}$ for $10 \mathrm{~min}$. Following blocking in $3 \%$ bovine serum albumin for $20 \mathrm{~min}$ at room temperature, the tissues were incubated overnight at room temperature with the following primary antibodies: $\alpha 7 \mathrm{nAChR}$ antibody (1:200; cat. no. sc-58607; Santa Cruz Biotechnology, Inc.) and anti-RNA binding protein 
Table I. Baseline characteristics.

\begin{tabular}{lccccc}
\hline & \multicolumn{5}{c}{ Groups } \\
\cline { 2 - 6 } Variables & Sham & CPR & US & US+MLA & GTS-21 \\
\hline Weight, $\mathrm{g}$ & $375.2 \pm 15.4$ & $378.3 \pm 16.8$ & $371.0 \pm 16.6$ & $367.0 \pm 15.9$ & $374.1 \pm 14.6$ \\
HR at baseline, bpm & $367 \pm 19$ & $371 \pm 22$ & $366 \pm 23$ & $359 \pm 18$ & $366 \pm 21$ \\
SBP, $\mathrm{mmHg}$ & $138.4 \pm 13.4$ & $139.8 \pm 14.2$ & $135.5 \pm 11.1$ & $133.4 \pm 9.1$ & $135.5 \pm 11.7$ \\
DBP, $\mathrm{mmHg}$ & $117.6 \pm 11.0$ & $124.4 \pm 11.8$ & $125.3 \pm 8.3$ & $117.9 \pm 9.8$ & $123.9 \pm 11.3$ \\
MAP at baseline, $\mathrm{mmHg}$ & $124.8 \pm 10.9$ & $129.5 \pm 11.9$ & $129.0 \pm 8.6$ & $123.0 \pm 8.8$ & $127.9 \pm 10.7$ \\
Preparation time, min & $44.0 \pm 1.1$ & $43.0 \pm 0.8$ & $43.2 \pm 0.8$ & $43.2 \pm 1.0$ & $44.0 \pm 1.0$ \\
Temperature at baseline, ${ }^{\circ} \mathrm{C}$ & $37.0 \pm 0.1$ & $36.9 \pm 0.0$ & $36.9 \pm 0.1$ & $37.0 \pm 0.1$ & $37.0 \pm 0.0$ \\
\hline
\end{tabular}

CPR, cardiopulmonary resuscitation; US, ultrasound; HR, heart rate; SBP, systolic blood pressure; DBP, diastolic blood pressure; MAP, mean arterial pressure.

fox-1 homolog $3(\mathrm{NeuN})$ rabbit polyclonal antibody $(1: 1,000$; cat. no. GB11138; Servicebio Biotechnology, Inc.). Following washing 3 times with PBS, sections were incubated for $1 \mathrm{~h}$ at room temperature with Cy3-labeled goat anti-rat IgG (1:200; cat. no. GB22302; Servicebio Biotechnology, Inc.) and fluorescein isothiocyanate-labeled goat-anti rabbit $\operatorname{IgG}$ (1:200; cat. no. GB22303; Servicebio Biotechnology, Inc.). The sections were subsequently mounted in Mowiol mounting medium (cat. no. 81381, Sigma-Aldrich; Merck KGaA) containing $1 \mu \mathrm{g} / \mathrm{ml}$ DAPI for DNA staining. Finally, images were captured using a laser scanning confocal microscope (Olympus FV3000; Olympus Corporation) at magnification, x200.

Western blot analysis. The hippocampal tissues were homogenized in a lysis buffer (Beyotime Institute of Biotechnology Co., Ltd.) and were placed on ice and incubated for $30 \mathrm{~min}$. The homogenates were centrifuged at $12,000 \mathrm{x} \mathrm{g}$ at $4^{\circ} \mathrm{C}$ for $10 \mathrm{~min}$ to separate the supernatant. The proteins were quantified by using the BCA kit (Servicebio Biotechnology, Inc.). A total of $40 \mu \mathrm{g}$ protein per lane was separated by $10 \%$ SDS-PAGE and transferred to polyvinylidene fluoride membranes. The membranes were blocked for $2 \mathrm{~h}$ at room temperature with blocking buffer and then incubated with primary antibodies targeting $\alpha 7 \mathrm{nAChR}$ (1:500; cat. no. sc-58607; Santa Cruz Biotechnology, Inc.) or GAPDH (1:1,000; cat. no. AS1039; Aspen, Wuhan, China) at $4^{\circ} \mathrm{C}$ overnight. The membranes were washed with TBS containing $0.05 \%$ Tween-20, and membranes were incubated with horseradish peroxidase-conjugated secondary antibodies against $\alpha 7 \mathrm{nAChR}$ (cat. no. GB23302; 1:3,000; Servicebio Biotechnology, Inc.) and GADPH (AS1107; 1:5,000; Aspen) for $1 \mathrm{~h}$ at room temperature. The bands were developed using enhanced chemiluminescence (Beyotime Institute of Biotechnology, Co., Ltd.) and images were obtained using a Bio-Image Analysis System (Bio-Rad Laboratories, Inc.). Densitometric analysis was performed with ImageJ v1.49 (National Institutes of Health).

Statistical analysis. Continuous variables are presented as mean \pm standard deviation when data was normally distributed, or as a median (with 25 and 75th percentiles) when data was not normally distributed. Normal distribution was confirmed with the Kolmogorov-Smirnov test. Variables were compared with one-way analysis of variance, or the Kruskal-Wallis test for nonparametric data. If there was a significant difference in the overall comparison of the groups, comparisons between groups were made using the Bonferroni post-hoc test. Survival data for Kaplan-Meier curves were examined using the log-rank test. $\mathrm{P}<0.05$ was considered to be statistically significant.

\section{Results}

US preconditioning decreases the number of defibrillations required and shortens the duration of CPR. The present study initially used a total of 45 rats; however, 5 were excluded due to instrumental or technical failure during animal preparation. Therefore, 40 rats were randomly assigned to one of five groups ( $n=8 /$ group). No differences in basic parameters among the 5 groups were detected at baseline (Table I). During CPR, the aortic diastolic pressure was maintained at an even level, and no significant difference was observed among the resuscitation groups (Fig. 2A). US also did not alter the heart rates of rats when compared with those in CPR, MLA and GTS groups during resuscitation (Fig. 2B). However, US markedly decreased the number of defibrillations required and shortened the duration of CPR when compared with the CPR group (Fig. 2C and D). However, inhibition of $\alpha 7 \mathrm{nAChR}$ by MLA decreased the protective effects of US, as there was no significant difference between the CPR and MLA groups. Compared with the US group, GTS-21 treatment had a similar effect in decreasing the number of defibrillations required and the duration of CPR.

US suppresses the serum levels of TNF- $\alpha$ and IL- 6 and improves the $72 \mathrm{~h}$ survival rate following ROSC. An increase in circulating inflammatory cytokines was observed in all animals following resuscitation when compared with the SHAM group. The levels of TNF- $\alpha$ and IL-6 in the serum reached their peak at $4 \mathrm{~h}$ following the ROSC. There was a significant decrease in the levels of TNF- $\alpha$ and IL- 6 in the US group when compared with the CPR group, indicating that US may suppress the inflammatory response following 
A
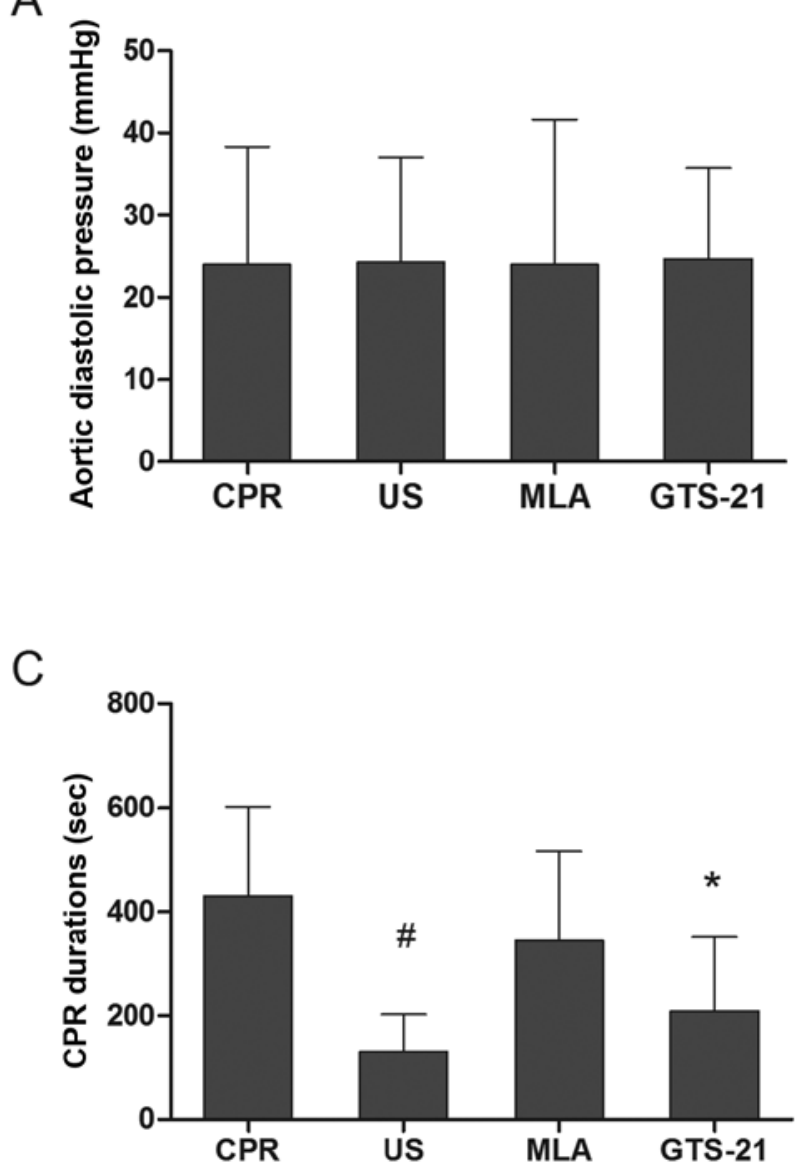

B

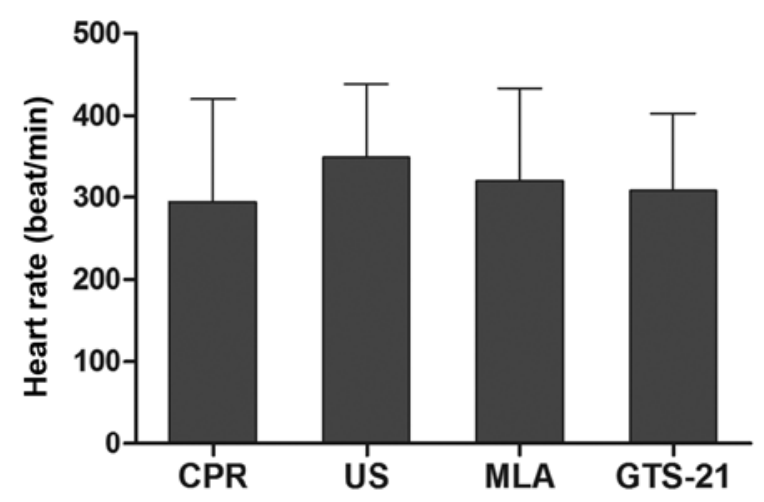

D

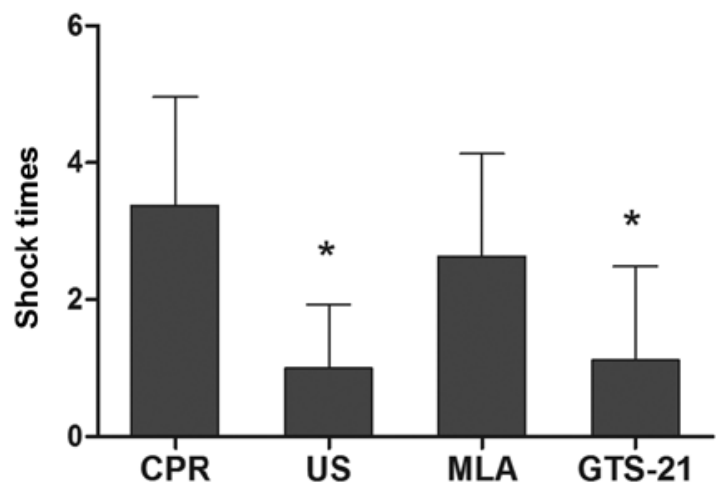

Figure 2. No significant difference in (A) aortic diastolic pressure and (B) heart rates between all groups during CPR were observed. However, the decrease in (C) CPR duration and (D) electrical shock times was observed in US-treated animals. " $\mathrm{P}<0.01$ vs. CPR group; ${ }^{\mathrm{P}}<0.05$ vs. CPR group. US, Ultrasound; $\mathrm{CPR}$, cardiopulmonary resuscitation.

resuscitation (Fig. 3A and B). In addition, an improved $72 \mathrm{~h}$ survival was observed in the US group when compared with the CPR group (Fig. 3C). The inhibition of $\alpha 7 \mathrm{nAChR}$ resulted in increased levels of TNF- $\alpha$ and IL-6 in MLA-treated animals. In the GTS group, the levels of inflammatory cytokines were similar to that of the US group, thereby demonstrating that the nicotinic receptor was associated with the protective effect of USA.

US improves NDS following resuscitation. Daily evaluations of the NDS revealed that less neurofunctional impairment occurred in animals in the US group when compared with those in the CPR group at 24 and 48 h post-ROSC (Fig. 3). At $72 \mathrm{~h}$ following resuscitation there was still a trend towards improved neurological function in the US group, yet the differences between the US and CPR groups were not statistically significant. There was no significant difference between the CPR and GTS groups, although the NDS mean value of the former group was larger compared with the latter group (Fig. 4). The inhibition of $\alpha 7 \mathrm{nAChR}$ by MLA abrogated the neuroprotective effect of US treatment, as evidenced by the larger NDS in the MLA group following ROSC.

US attenuates CPR-induced injury to hippocampal neurons. The photomicrographs of hippocampal neurons revealed that CPR resulted in an increase in the amount of darkly stained smaller nuclei and neurons with vacuolization of the cytoplasm in the hippocampal CA1 region at $72 \mathrm{~h}$ after ROSC, which suggested that a large amount of neuronal mortality had occurred in the hippocampal CA1 region (Fig. 5A). However, the aforementioned neuronal changes were less severe in the US and GTS groups in comparison with CPR groups, indicating that US treatment and GTS-21 exerted similar neuroprotective effects. The inhibition of $\alpha 7 \mathrm{nAChR}$ by MLA abrogated the neuroprotective effect of US treatment, as evidenced by an increase in the amount of neuronal death in the CA1 region (Fig. 5B).

US increases the expression of $\alpha 7 n A C h R$ in hippocampal neurons following CPR. As demonstrated in Fig. 6, a marked decrease in the number of $\alpha 7 \mathrm{nAChR}$-positive cells co-located in the neuron was observed visually in the CA1 region of the hippocampus in the CPR group compared with the SHAM group. However, US pretreatment reversed the decrease in $\alpha 7 n$ AChR levels caused by CPR, suggesting that the central cholinergic signaling may be repaired by US following resuscitation (Fig. 6A). In addition, no significant difference was identified between the MLA and CPR groups. However, GTS-21 had a similar effect as US treatment. The western blot analysis results of $\alpha 7 \mathrm{nAChR}$ protein expression were consistent with the immunofluorescence analysis data (Fig. 6B and C). 
A

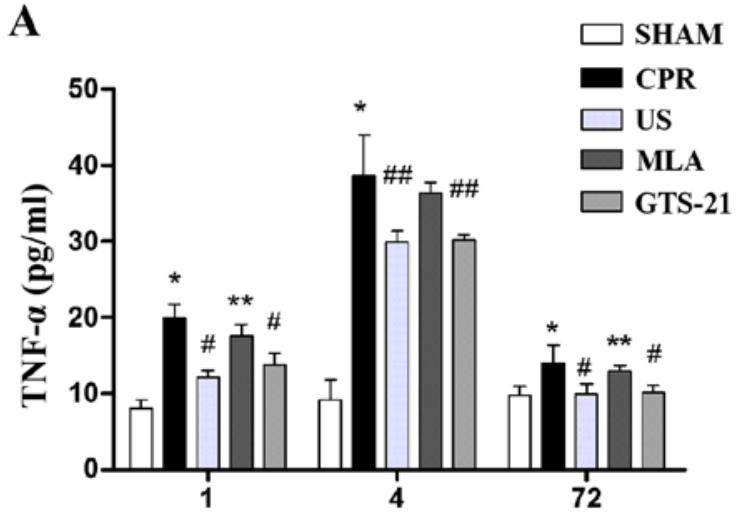

Time (h)

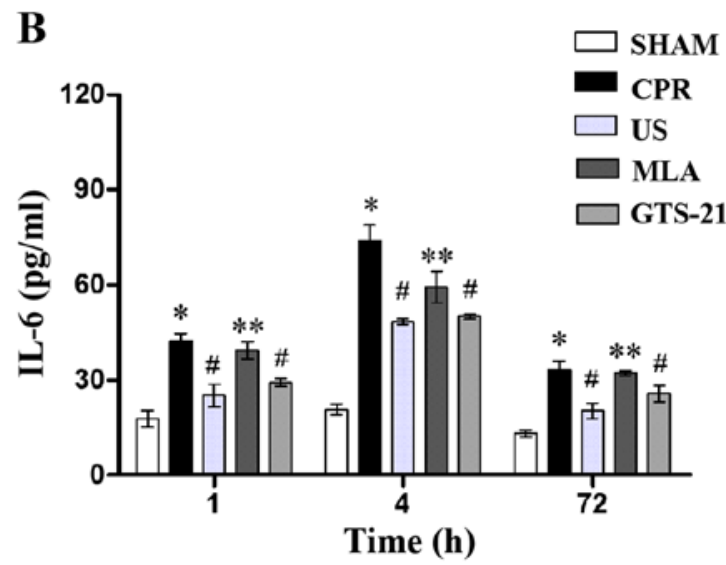

C

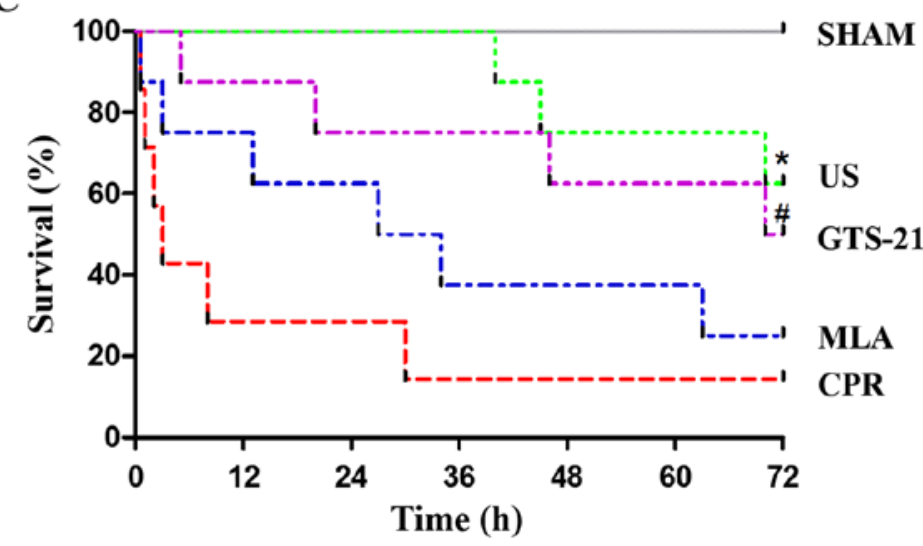

Figure 3. US inhibits the concentrations of (A) TNF- $\alpha$ and (B) IL-6 at 1,4 and $72 \mathrm{~h}$ following resuscitation. ${ }^{*} \mathrm{P}<0.01$ vs. SHAM. ${ }^{* *} \mathrm{P}<0.01$ vs. US; ${ }^{\#} \mathrm{P}<0.01$ and ${ }^{\# \#} \mathrm{P}<0.05$ vs. CPR. (C) US prolonged the survival time following CPR. Kaplan-Meier curves of the cumulative survival rates at $72 \mathrm{~h}$ following resuscitation. ${ }^{*} \mathrm{P}<0.01$ US vs. CPR; ${ }^{\mathrm{P}}<0.05$ GTS-21 vs. CPR. US, ultrasound; CPR, cardiopulmonary resuscitation; TNF- $\alpha$, tumor necrosis factor- $\alpha$; IL-6, interleukin-6.

\section{Discussion}

The results of the present study clearly demonstrated that the survival rate was significantly improved when rats were exposed to a single dose of US at $24 \mathrm{~h}$ prior to CPR. In addition, US treatment decreased the number of electrical shocks required for ROSC, shortened the duration of CPR and attenuated neurological dysfunction. It was also observed that the levels of circulating inflammatory cytokines were suppressed following resuscitation and the expression of $\alpha 7 \mathrm{nAChR}$ was promoted in hippocampal neurons by US treatment. It was also observed that the inhibition of $\alpha 7 \mathrm{nAChR}$ by MLA abrogated the protective effect of US, suggesting that ultrasonic protection against IRI resulted from CPR was strongly dependent on the activation of CAP via $\alpha 7 n A C h R$.

It has previously been demonstrated that mice that had been subjected to US $24 \mathrm{~h}$ prior to IRI were protected against acute kidney injury (AKI) (13). Although the role of US in kidney IRI has been investigated, the protective effects of US during global IRI resulting from CA/CPR have yet to be completely elucidated. Therefore, the aim of the present study was to observe if US prior to VF would improve patient outcomes following CPR.

Inflammatory cytokines have been implicated in the pathophysiology of post-cardiac arrest syndrome, which include myocardial dysfunction and hypotension, often leading to multi-organ system dysfunction and mortality. The results of

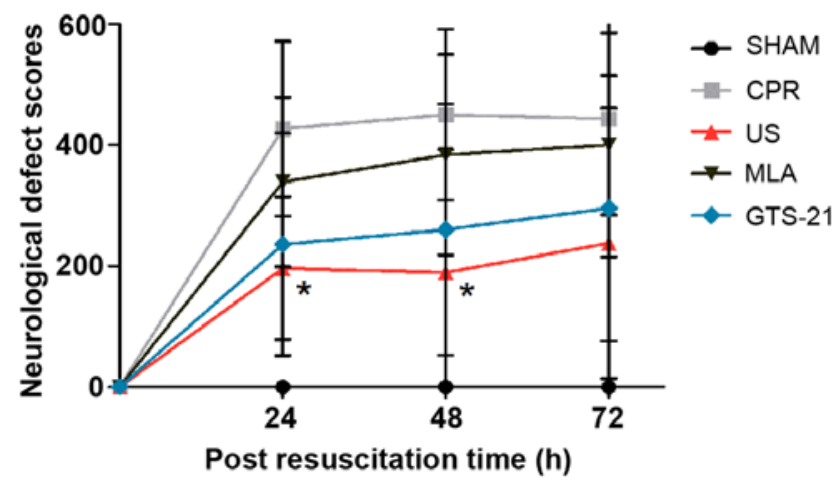

Figure 4. Neurological Defect Score at 24,48 and $72 \mathrm{~h}$ following resuscitation in all groups. The neurologic deficits were scored from 0 (no observed neurologic deficit) to 500 (death or brain death). ${ }^{*} \mathrm{P}<0.05$ US vs. CPR. US, ultrasound; CPR, cardiopulmonary resuscitation.

the present study indicated that receiving US $24 \mathrm{~h}$ prior to CPR prevented the increase in TNF- $\alpha$ and IL- 6 following resuscitation. TNF- $\alpha$ has been reported to be inversely correlated with myocardial function (14), and the administration of a monoclonal antibody directed against TNF- $\alpha$ attenuates myocardial dysfunction and improves short-term survival in the early post cardiac arrest period (15). IL-6 in cerebrospinal fluid and blood have been indicated to be correlated with neurological outcome following CA (16). It has also been demonstrated that 
A
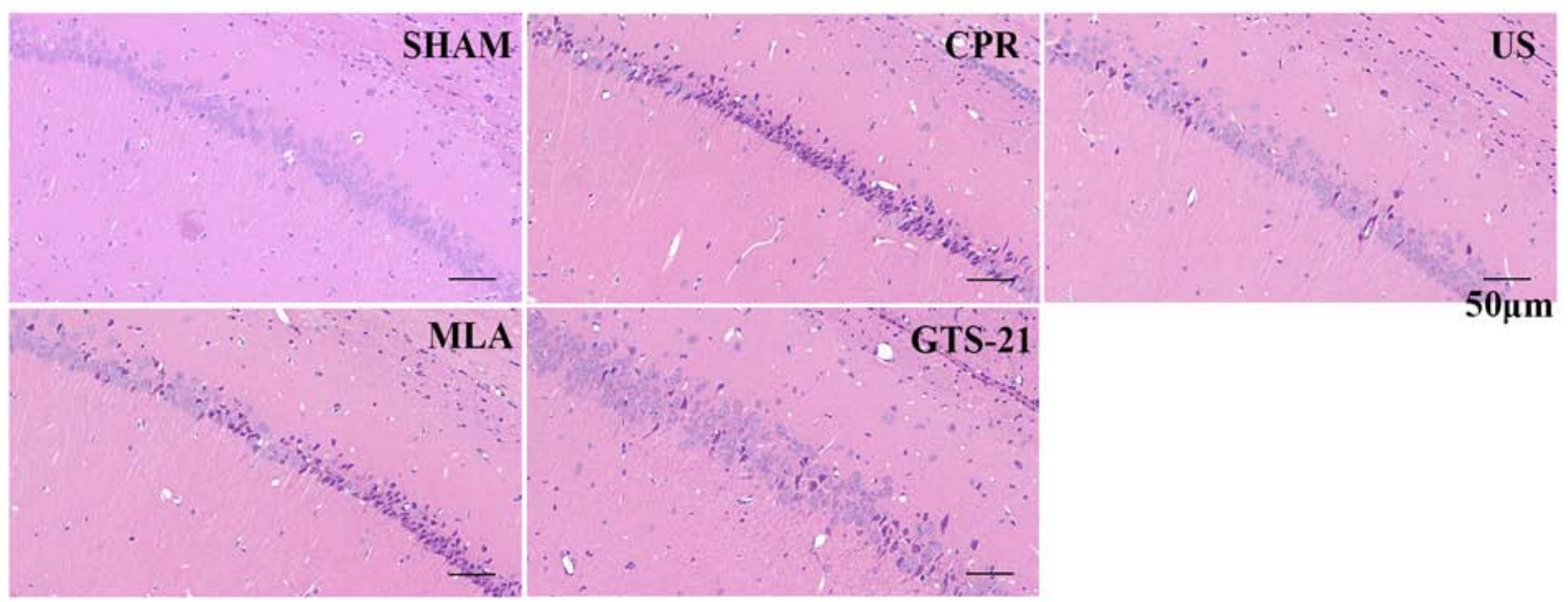

$50 \mu \mathrm{m}$

B

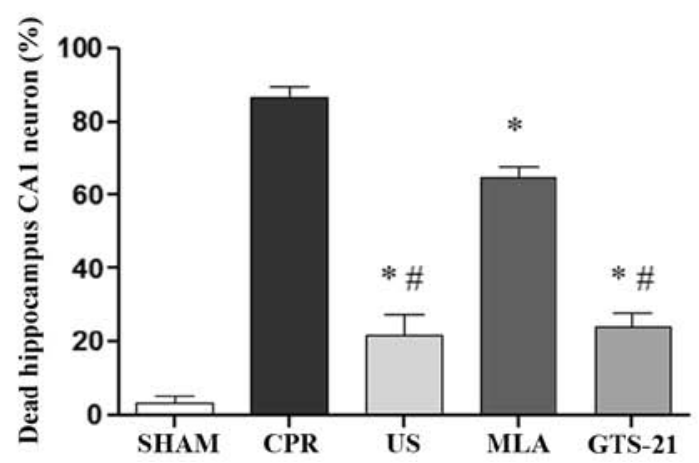

Figure 5. Hippocampal morphology as assessed in hematoxylin \& eosin-stained tissue sections. (A) The prevention of the injury to hippocampal neurons in animals exposed to US treatment was confirmed. Scale bar, $50 \mu \mathrm{m}$. (B) Quantification of hippocampal CA1 neuronal cell death. ${ }^{*} \mathrm{P}<0.01 \mathrm{vs.} \mathrm{CPR}$; ${ }^{\#} \mathrm{P}<0.01$ vs. MLA. US, ultrasound; CPR, cardiopulmonary resuscitation.

IL-6 values on intensive care unit admission are associated with increased mortality in out-hospital patients with CA (17). Therefore, it is reasonable to conclude that the inhibition of TNF- $\alpha$ and IL- 6 by US could contribute to the improvement in survival following CPR. In addition, the anti-inflammatory effect of US treatment may depend on $\alpha 7 \mathrm{nAChR}$ as it was abolished by MLA and was imitated by GTS-21, suggesting that the protection provided by US prior to CPR is dependent on the CAP.

The present study also revealed that US may decrease the number of defibrillations required during CPR and shorten the CPR duration. The data demonstrated there was no difference in myocardial reperfusion during CPR, as evidenced by consistent aortic diastolic pressure among the CPR, US, MLA and GTS groups. A potential explanation for this result is the alterations in gap junction intercellular communication during resuscitation. Gap junctions are composed of connexins, predominantly connexin 43 , in the ventricle, and provide aqueous conduits between cells to allow electrical current flow (18). It has been demonstrated that the concentration of TNF- $\alpha$ is rapidly increased in rats following VF (19), and TNF- $\alpha$ may decrease the expression of connexin 43 in the heart (20), which is associated with gap junction uncoupling and results in increasing cardiac arrhythmias and impaired defibrillation. Regional gap junction uncoupling markedly increases the threshold values of biphasic defibrillations (21). Therefore, a potential explanation for the anti-arrhythmic effects of US may be due to its potential to inhibit gap junction uncoupling by suppressing the inflammatory cytokines during CPR. In the present study, MLA was observed to reverse the anti-arrhythmic effects of US treatment, whereas GTS-21 exerted anti-arrhythmic effects similar to US treatment, suggesting that the anti-arrhythmic effects of US was driven through the CAP via $\alpha 7 \mathrm{nAChR}$.

In addition to the aforementioned cardioprotective effects of US during resuscitation, the present study also demonstrated the neuroprotective effects of US. Brain injury is a common reason for morbidity and mortality following resuscitation, and the results of the present study supported the hypothesis that US may alleviate cerebral IRI following $\mathrm{CPR}$, as evidenced by the improvement in neurological deficits and the decreases in the severity of injury to hippocampal neurons following US treatment. Although MLA aggravated neurological dysfunction in US-treated animals, GTS-21 failed to attenuate neurological impairment following ROSC when compared with control animals, despite the presence of a trend toward improved neurological function in GTS-treated animals. A potential explanation for this result may be associated with the usage of GTS-21 in the present study. As GTS-21 has a biological half-life of 12-24 h (22), only one dose was delivered $30 \mathrm{~min}$ prior to $\mathrm{VF}$. The improved neurological outcomes may be observed if GTS-21 is administered twice a day over 3 consecutive days. In addition, it is well known that cerebral IRI largely depends on the duration of ischemia. As US treatment shortened the duration of CPR, these cardiac effects may also serve a role in the neuro-protection observed. 
A
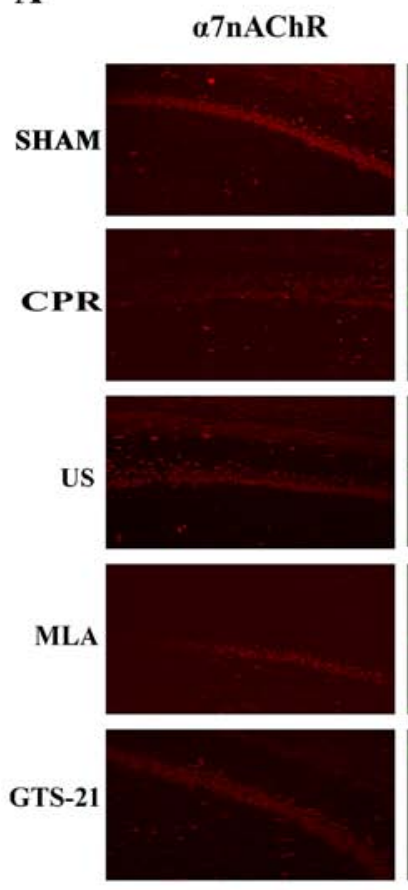

NeuN
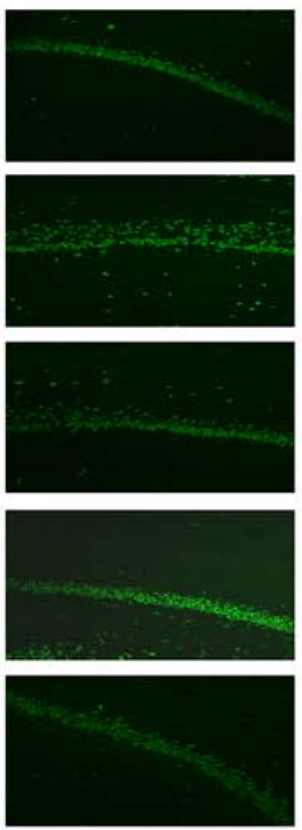

Merged
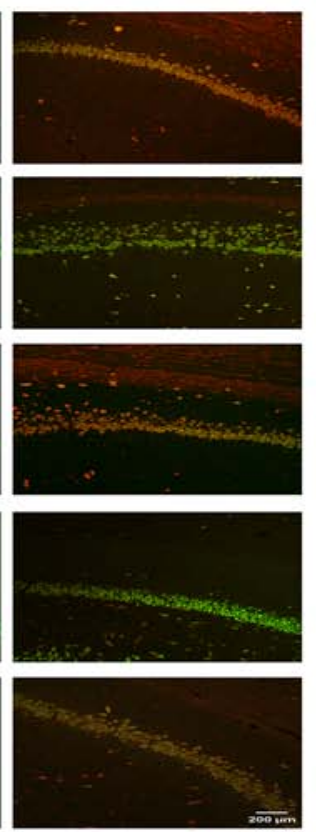

B

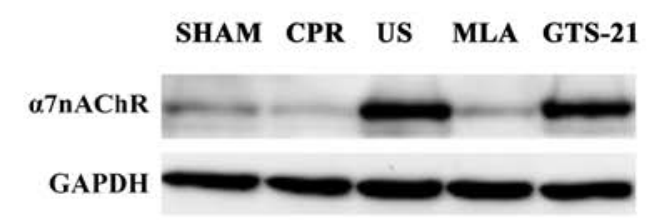

C

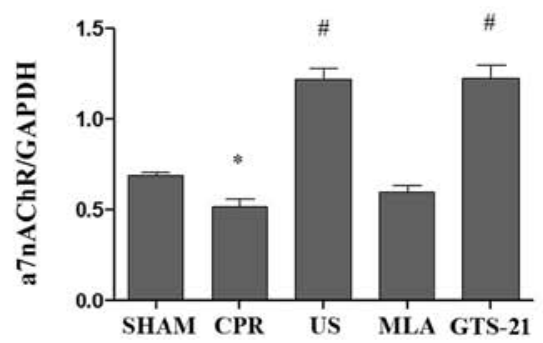

Figure 6. NeuN and $\alpha 7 \mathrm{nAChR}$ expression patterns. (A) Immunostaining for neuronal marker NeuN and $\alpha 7 \mathrm{nAChR}$. $\alpha 7 \mathrm{nAChR}$ was stained in red and NeuN was stained in green. The microphotograph representing the double staining for $\alpha 7 \mathrm{nAChR}$ and NeuN demonstrated that the hippocampal neurons expressed $\alpha 7 \mathrm{nAChR}$. Scale bar, $200 \mu \mathrm{m}$. (B) Representative blots of $\alpha 7 \mathrm{nAChR}$ proteins detected by western blot analysis. (C) The levels of $\alpha 7 \mathrm{nAChR}$ in hippocampus at $72 \mathrm{~h}$ following resuscitation. ${ }^{*} \mathrm{P}<0.05$ vs. SHAM group; ${ }^{\#} \mathrm{P}<0.01$ vs. CPR group. NeuN, anti-RNA binding protein fox-1 homolog 3 ; $\alpha 7 \mathrm{nAChR}, \alpha 7$ nicotinic acetylcholine receptor; US, ultrasound; CPR, cardiopulmonary resuscitation.

Furthermore, the present study examined whether the hippocampal neurons expressed $\alpha 7 \mathrm{nAChR}$ on their surface following treatment with US, which may suggest a potential involvement of the CAP in cerebral reperfusion injury. It has previously been demonstrated that CA and CPR dysregulates central cholinergic signaling and engenders large increases in neuroinflammation and neuronal damage (23). The results of the present study also demonstrated that the expression of $\alpha 7 \mathrm{nAChR}$ in the hippocampal CA1 region was decreased at $72 \mathrm{~h}$ following ROSC in the CPR group. However, in the US group, the hippocampal neurons exhibited high levels of $\alpha 7 \mathrm{nAChR}$ on their surface, indicating that US may restore the central cholinergic signaling against IRI induced by CPR. However, the contribution of US to the brain response against IRI via the CAP requires additional verification in future studies. US has been demonstrated to markedly increase the spike frequency of primary hippocampal neurons in culture (24). The increase in excitability is largely mediated by the mechanical effects of US, which may result in a mechanical distortion of cell membranes, and these mechanical deformations may cause the activation of mechanosensitive ion channels (25), which may alter the polarization state of neurons and cause upregulation or suppression in activity. US application to the left kidney activates the adrenergic splenic nerve, as the left kidney is closed to the spleen. We hypothesized that, in the present study, US activated the adrenergic splenic nerve resulting in the release of norepinephrine, which binds to adrenergic receptors on nearby $\mathrm{CD} 4+\mathrm{T}$ cells. This stimulates the production of acetylcholine, which binds to $\alpha 7 \mathrm{nAChRs}$ on splenic myeloid cells and results in decreased inflammation and IRI (8). However, the mechanism of the contribution of US treatment to the brain response against IRI via the CAP remains to be determined.

There were several limitations to the present study: Firstly, it is difficult to argue that the protective effects against whole-body IRI were as a result of US pretreatment in such a short time period ( $24 \mathrm{~h}$ ). However, it has been demonstrated that mice that had been subjected to US for 5 min 1 day prior to IRI were protected against AKI (8). Therefore, it is reasonable to interpret the results of the present study, as the same US application protocol described by Gigliotti et al (8) was used. In addition, US treatment should also be applied following $\mathrm{CA}$, in order to investigate whether this also exerts a beneficial effect. The spleen should also be investigated, as the spleen is the tissue target for US-mediated tissue protection (13), and therefore may be more effective. Additional studies are required in order to investigate the different conditions of US treatment. Secondly, in a clinical setting, patients treated with CPR typically have a pre-existing clinical disease; however, the animals used in the present study were healthy. Therefore, the outcome of this study in a rat model of CPR requires further verification in large animal and clinical studies.

In summary, the present study demonstrated that US exerted a remarkable ability to improve the clinical outcomes following $\mathrm{CPR}$, and the mechanism underlying the protection of US was associated with the activation of CAP via the a7nAChR. Importantly, the noninvasive US regimen relies on US settings within approved United States of America Food and Drug Administration guidelines, and has been used for the treatment of solid tumors, uterine fibroids, glaucoma, kidney stones, deep venous thrombosis and musculoskeletal injuries (26-28). In addition, the US method used in the present study was simple 
and may be administered by a routine clinical imaging system, which is suitable for emergency operation. Therefore, this non-pharmacological approach, involving therapeutic US within the spectrum of use currently approved for humans, provides an attractive alternative therapy for post-resuscitation syndrome.

\section{Acknowledgements}

Professor Wei Peng in the University of Utah School of Medicine contributed to the editing of this manuscript. The abstract was presented at Resuscitation Science Symposium 2018 Nov 10-11 in Chicago, IL and published as abstract no. 254 in Circulation 138 (Suppl 2): 2018.

\section{Funding}

The present study was supported by the National Nature Science Foundation of China (grant no. 81571866) and Health Commission of Hubei Province (grant no. WJ2019M160).

\section{Availability of data and materials}

The datasets used and analyzed in the present study are available from the corresponding author on reasonable request.

\section{Authors' contributions}

YZ, YS, LG and PS contributed to the conception and design of research. YZ, YS, TS, LL and WS performed the experiments. YZ, YS, TS, LL, WS and PS analyzed data. YZ, YS, TS, LL, WS and PS interpreted results of the experiments. YZ, YS and TS prepared figures/ YZ, YS, TS, LL, WS and PS the drafted manuscript. YZ, YS, TS, LG and PS edited and revised manuscript. PS approved the final version of manuscript. All authors read and approved the final manuscript.

\section{Ethics approval and consent to participate}

All protocols in the animal model were conducted in strict accordance with the guidelines of the Ministry of Science and Technology of the People's Republic of China for Animal Care and Use, which conformed to the Guide for the Care and Use of Laboratory Animals published by the United States of America National Institutes of Health (9). The study was approved by the Medical Ethics Committee of Huazhong University of Science and Technology (approval no. 2017-S2191).

\section{Patient consent for publication}

Not applicable.

\section{Competing interests}

The authors declare that they have no competing interests.

\section{References}

1. Adrie C, Adib-Conquy M, Laurent I, Monchi M, Vinsonneau C Fitting C, Fraisse F, Dinh-Xuan AT, Carli P, Spaulding C, et al: Successful cardiopulmonary resuscitation after cardiac arrest as a 'sepsis-like' syndrome. Circulation 106: 562-568, 2002.
2. Borovikova LV, Ivanova S, Zhang M, Yang H, Botchkina GI, Watkins LR, Wang H, Abumrad N, Eaton JW and Tracey KJ: Vagus nerve stimulation attenuates the systemic inflammatory response to endotoxin. Nature 405: 458-462, 2000.

3. Zhao M, He X, Bi XY, Yu XJ, Gil Wier W and Zang WJ: Vagal stimulation triggers peripheral vascular protection through the cholinergic anti-inflammatory pathway in a rat model of myocardial ischemia/reperfusion. Basic Res Cardiol 108: 345, 2013.

4. Sun Z, Baker W, Hiraki T and Greenberg JH: The effect of right vagus nerve stimulation on focal cerebral ischemia: An experimental study in the rat. Brain Stimul 5: 1-10, 2012.

5. Inoue T, Abe C, Sung SS, Moscalu S, Jankowski J, Huang L, Ye H, Rosin DL, Guyenet PG and Okusa MD: Vagus nerve stimulation mediates protection from kidney ischemia-reperfusion injury through $\alpha 7 \mathrm{nAChR}^{+}$plenocytes. J Clin Invest 126: 1939-1952, 2016.

6. Sun P, Wang J, Zhao S, Yang Z, Tang Z, Ravindra N, Bradley J, Ornato JP, Peberdy MA and Tang W: Improved outcomes of cardiopulmonary resuscitation in rats treated with vagus nerve stimulation and its potential mechanism. Shock 49: 698-703, 2018.

7. Hougardy JM, Sadis C and Le Moine A: Ultrasonic stimulation of the cholinergic anti-inflammatory pathway for renal protection. J Am Soc Nephrol 24: 1340-1342, 2013.

8. Gigliotti JC, Huang L, Ye H, Bajwa A, Chattrabhuti K, Lee S, Klibanov AL, Kalantari K, Rosin DL and Okusa MD: Ultrasound prevents renal ischemia-reperfusion injury by stimulating the splenic cholinergic anti-inflammatory pathway. J Am Soc Nephrol 24: 1451-1460, 2013.

9. Research NRCU: Guide for the Care and Use of Laboratory Animals. National Academies Press, Washington, DC, 1996.

10. Lin JY, Liao XX, Li H, Wei HY, Liu R, Hu CL, Huang GQ, Dai G and Li X: Model of cardiac arrest in rats by transcutaneous electrical epicardium stimulation. Resuscitation 81: 1197-1204, 2010.

11. Gong P, Zhao S, Wang J, Yang Z, Qian J, Wu X, Cahoon J and Tang W: Mild hypothermia preserves cerebral cortex microcirculation after resuscitation in a rat model of cardiac arrest. Resuscitation 97: 109-114, 2015.

12. Ye S, Weng Y, Sun S, Chen W, Wu X, Li Z, Weil MH and Tang W: Comparison of the durations of mild therapeutic hypothermia on outcome after cardiopulmonary resuscitation in the rat. Circulation 125: 123-129, 2012.

13. Gigliotti JC, Huang L, Bajwa A, Ye H, Mace EH, Hossack JA, Kalantari K, Inoue T, Rosin DL and Okusa MD: Ultrasound modulates the splenic neuroimmune axis in attenuating AKI. J Am Soc Nephrol 26: 2470-2481, 2015.

14. Niemann JT, Garner D and Lewis RJ: Tumor necrosis factor-alpha is associated with early postresuscitation myocardial dysfunction. Crit Care Med 32: 1753-1758, 2004.

15. Niemann JT, Youngquist ST, Shah AP, Thomas JL and Rosborough JP: TNF- $\alpha$ blockade improves early postresuscitation survival and hemodynamics in a swine model of ischemic ventricular fibrillation. Resuscitation 84: 103-107, 2013.

16. Oda Y, Tsuruta R, Kasaoka S, Inoue T and Maekawa T: The cutoff values of intrathecal interleukin 8 and 6 for predicting the neurological outcome in cardiac arrest victims. Resuscitation 80 : 189-193, 2009.

17. Vaahersalo J, Skrifvars MB, Pulkki K, Stridsberg M, Røsjø H, Hovilehto S, Tiainen M, Varpula T, Pettilä V and Ruokonen E; FINNRESUSCI Laboratory Study Group: Admission interleukin-6 is associated with post resuscitation organ dysfunction and predicts long-term neurological outcome after out-of-hospital ventricular fibrillation. Resuscitation 85: 1573-1579, 2014.

18. Van Kempen MJ, Fromaget C, Gros D, Moorman AF and Lamers WH: Spatial distribution of connexin 43, the major cardiac gap junction protein, in the developing and adult rat heart. Circ Res 68: 1638-1651, 1991.

19. Niemann JT, Rosborough J, Youngquist S, Lewis RJ, Phan QT and Filler S: The proinflammatory cytokine response following resuscitation in the swine model depends on the method of ventricular fibrillation induction. Acad Emerg Med 15: 939-944, 2008.

20. Fernandez-Cobo M, Gingalewski C, Drujan D and De Maio A: Downregulation of connexin 43 gene expression in rat heart during inflammation. The role of tumour necrosis factor. Cytokine 11: 216-224, 1999.

21. Sims JJ, Schoff KL, Loeb JM and Wiegert NA: Regional gap junction inhibition increases defibrillation thresholds. Am J Physiol Heart Circ Physiol 285: H10-H16, 2003. 
22. Pavlov VA, Ochani M, Yang LH, Gallowitsch-Puerta M, Ochani K, Lin X, Levi J, Parrish WR, Rosas-Ballina M, Czura CJ, et al: Selective a7-nicotinic acetylcholine receptor agonist GTS-21 improves survival in murine endotoxemia and severe sepsis. Crit Care Med 35: 1139-1144, 2007.

23. Norman GJ, Morris JS, Karelina K, Weil ZM, Zhang N, Al-Abed Y, Brothers HM, Wenk GL, Pavlov VA, Tracey KJ and Devries AC: Cardiopulmonary arrest and resuscitation disrupts cholinergic anti-inflammatory processes: A role for cholinergic a7 nicotinic receptors. J Neurosci 31: 3446-3452, 2011.

24. Khraiche ML, Phillips WB, Jackson N and Muthuswamy J: Ultrasound induced increase in excitability of single neurons. Conf Proc IEEE Eng Med Biol Soc 2008: 4246-4249, 2008.

25. Mihran RT, Lineaweaver S, Barnes FS and Wachtel H: Effects of pulsed acoustic and mechanical stimuli on the excitability of isolated neuronal and cardiac cells. Appl Occup Environ. Hyg 11: 271-274, 1996.
26. Lu T, Loh TM, El-Sayed HF and Davies MG: Single-center retrospective review of ultrasound-accelerated versus traditional catheter-directed thrombolysis for acute lower extremity deep venous thrombosis. Vascular 25: 525-532, 2017.

27. Nazer B, Gerstenfeld EP, Hata A, Crum LA and Matula TJ: Cardiovascular applications of therapeutic ultrasound. J Interv Card Electrophysiol 39: 287-294, 2014.

28. Miller DL, Smith NB, Bailey MR, Czarnota GJ, Hynynen K and Makin IR; Bioeffects Committee of the American Institute of Ultrasound in Medicine: Overview of therapeutic ultrasound applications and safety considerations. J Ultrasound Med 31: 623-634, 2012.

(i)(3) This work is licensed under a Creative Commons Attribution-NonCommercial-NoDerivatives 4.0 International (CC BY-NC-ND 4.0) License. 